\title{
Robot-assisted lung-reserved bronchial glomus tumor resection: a case report
}

\author{
Zhipeng Hao, Ni Zhang \\ Department of Thoracic Surgery, Tongji Hospital, Tongji Medical College, Huazhong University of Science and Technology, Wuhan, China \\ Correspondence to: Ni Zhang. Department of Thoracic Surgery, Tongji Hospital, Tongji Medical College, Huazhong University of Science and \\ Technology, Wuhan, China. Email: zhangnidoc@vip.163.com.
}

\begin{abstract}
Glomus tumors are benign mesenchymal neoplasm with an estimated incidence of $1.6 \%$ in 500 consecutive soft tissue tumors which usually located in the skin or soft tissue, respiratory tract was seldom involved. The majority of bronchial glomus tumor were treated by surgery, however, treated by robotic surgery has rarely been described. A 31-year-old woman was diagnosed to have a nodule located in the right main bronchus during chest CT exam without any symptoms, further bronchoscopy revealed the lesion was located at the root of the right main bronchus. The nodule needs to be removal by surgery, and also the pulmonary function needs to be preserved as much as possible. A robot-assisted surgery was then performed, and the "longitudinal resection with transverse suture" technique was adopted to avoid bronchial stenosis, postoperative pathology confirmed a bronchial glomus tumor. The patient was discharged 15 days after the operation. The anastomose healed well without stenosis when examined by bronchoscopy 30 days after surgery. Bronchial glomus tumor could be safely treated by robot-assisted lung-reserved surgery could be used safely to treat bronchial glomus tumor under suitable conditions (e.g., location, size of tumor), longitudinal resection with transverse suture could be used as an effective method to prevent bronchial stenosis.
\end{abstract}

Keywords: Case report; glomus tumor; robot-assisted surgery; lung-reserved

Received: 03 July 2021; Accepted: 18 October 2021; Published: 30 January 2022.

doi: $10.21037 /$ shc-21-16

View this article at: https://dx.doi.org/10.21037/shc-21-16

\section{Introduction}

Glomus tumor originate from glomus apparatus and is a rare neoplasm that accounts for $1.6 \%$ of soft tissue tumors (1). The dermis and subcutaneous tissue are the most common location of glomus tumor, the occurrence in the bronchus is extremely rare $(2,3)$. Sleeve resection with primary reconstruction of bronchus and endoscopic removal are usually used for the treatment of bronchial glomus tumors $(4,5)$. Robot-assisted bronchoplasty has been developed for curative treatment of lung cancer to avoid pneumonectomy (6), however, no literature had reported the treat of bronchial glomus tumor by robot-assisted surgery using "longitudinal resection with transverse suture" technique. We present a case of a 31-year-old woman with glomus tumor at the root of the right main bronchus. The tumor was successfully removed by robot-assisted surgery without pulmonary resection (lung-reserved) and no bronchostenosis was observed during bronchoscopy 30 days after surgery. Robotic surgery may be expected to be one of the options for bronchial glomus tumors. We present the following article in accordance with the CARE reporting checklist (available at https://shc.amegroups.com/article/ view/10.21037/shc-21-16/rc).

\section{Case presentation}

A 31-year-old woman who underwent chest CT examination during routine physical examination was found to have a nodule in the right bronchus with no symptoms such as cough, sputum, hemoptysis, or wheezing. No comorbidity was found. 

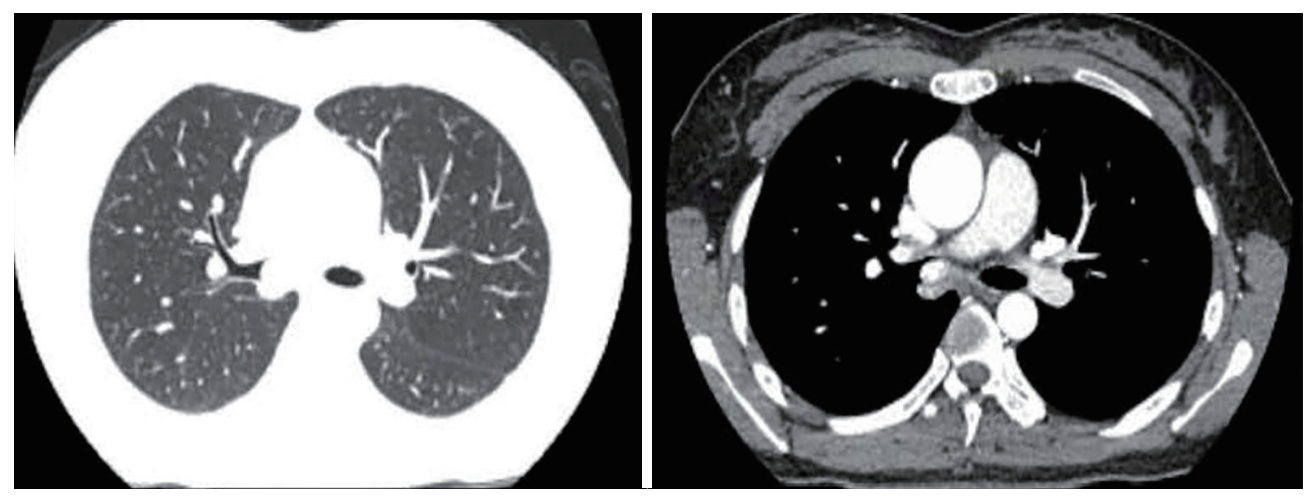

Figure 1 CT scan showed the nodule at the end of the right main bronchus.

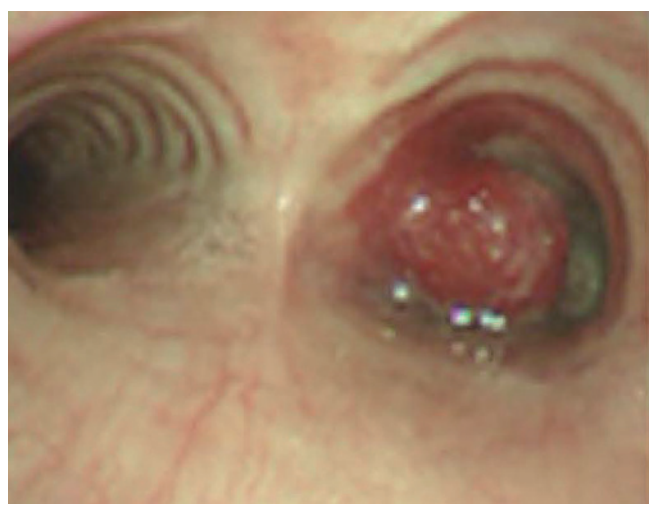

Figure 2 Bronchoscopy revealed a new organism at the root of the right main bronchus with obvious congestion.

Chest CT scan showed the nodule was located in the right main bronchus (Figure 1). Further bronchoscopy revealed a new organism at the root of the right main bronchus with obvious congestion (Figure 2). Considered the risk of bleeding, biopsy was not conducted.

After detailed evaluation, we decided to resect the lesion under robotic surgery to minimize surgical trauma without pulmonary resection. During the operation, the patient was placed in the left lateral decubitus position, the operating table was flexed to maximize the intercostal spaces. General anesthesia was induced and intubation was achieved via a double lumen endotracheal tube.

The observation hole was made at the 8 th intercostal space at the posterior axillary line, and the operation holes are the 6th intercostal space at the scapular line and the anterior axillary line and the 8 th intercostal space at the mid-axillary line.

The operation time was $118 \mathrm{~min}$, and the blood lose was less than $100 \mathrm{~mL}$. During the operation, the posterior mediastinal pleura were first opened to sufficiently reveal the right bronchus. It was clear that the tumor located at the bronchial membrane. The posterior mediastinal pleura were suspended to the posterior chest wall in order to fully expose the surgical field. The lymph nodes under the carina were thoroughly cleaned, and the carina, the right intermedius bronchus and the right upper bronchus should be dissociated as much as possible to obtain sufficient resection and anastomosis distance. Because the blood supply of the nodule is relatively abundant, the bronchial artery around the nodule should be ligatured as much as possible before resection in order to reduce the bleeding risk. During tumor resection, intraoperative margin excision and frozen section analysis were performed, we expanded the resection range twice to make sure the margins was negative.

Due to the large size of the resected area, we adopt the method of "longitudinal resection with transverse suture", the long diameter of the resection was parallel to the bronchus direction, and the suture direction was perpendicular to the long diameter. This method may effectively avoid the bronchial stenosis after anastomosis. Continuous suture with a 4-0 Surgipro suture was used, after the continuous anastomosis is completed, 3-0 Vicryl suture was used to reinforce the membrane. After the anastomosis was completed, the right lung was well recruited when the lung was expanded (Video 1).

Antibiotics and anti-coagulation are administered during post-operative management, patient was encouraged to cough properly and get out of bed as soon as possible. The chest X-ray exam was conducted on the 4th day after the operation, and showed the right lung re-expanded well, the chest tube was then removed. She was discharged home well on post-operative day 15 , and chest CT showed that the 


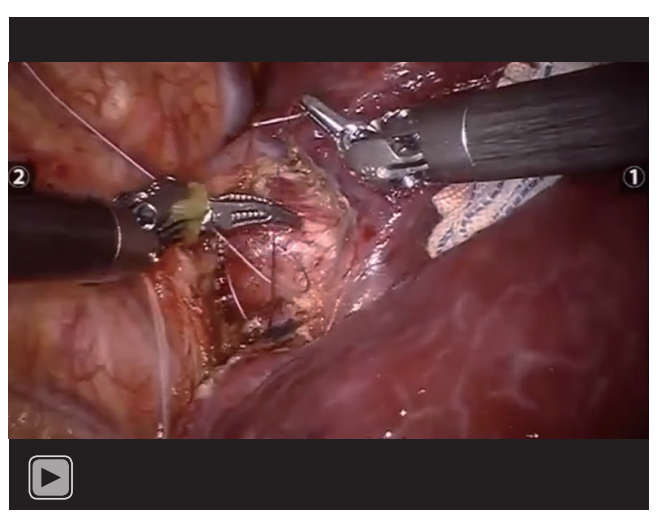

Video 1 Robotic surgery for bronchial glomus tumor.

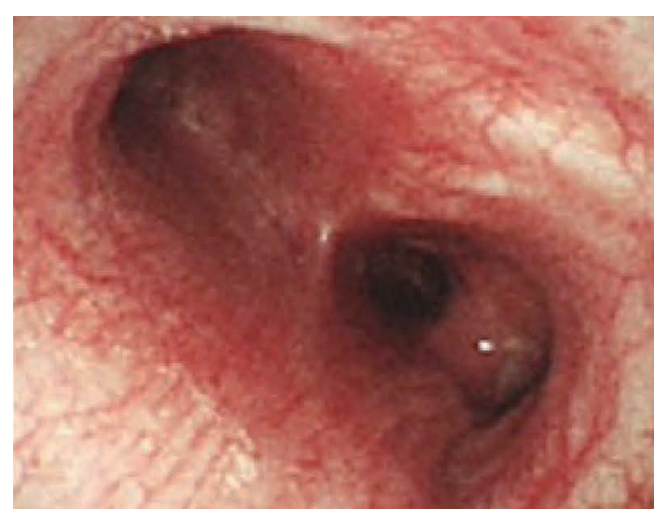

Figure 4 Bronchoscopy showed the carina on post-operative day 30 .
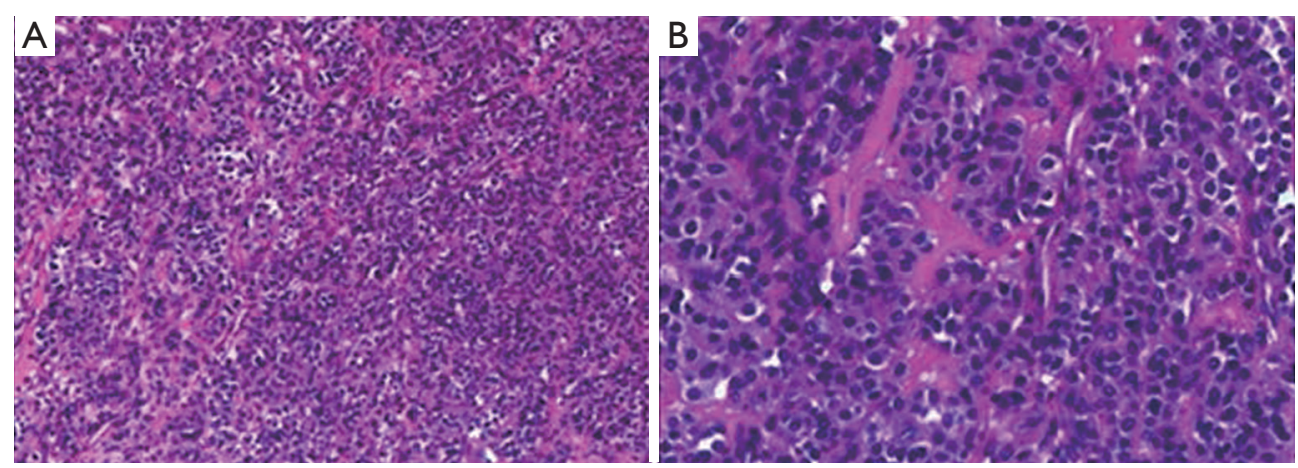

Figure 3 Histopathologic specimen. (A) HE staining; magnification, $\times 100$; (B) HE staining; magnification, $\times 400$.

right bronchus was unobstructed and the right pulmonary was recruited well. Postoperative pathology analysis of the tumor indicated a bronchial glomus tumor with negative margins (Figure 3). Bronchoscopy on post-operative day 30 also showed that the right bronchus cavity was clear and the anastomose was not stenosis (Figure 4). During the 2-year follow-up, patient complained no cough, hemoptysis and dyspnea, all her life changed nothing. All procedures performed in studies involving human participants were in accordance with the ethical standards of the institutional research committee and with the Helsinki Declaration (as revised in 2013). Written informed consent was obtained from the patient for publication of this case report and accompanying images. A copy of the written consent is available for review by the editorial office of this journal.

\section{Discussion}

Glomus tumor is a rare neoplasm especially in bronchus, the primary treatments consisted of endoscopic resection and surgical resection (including segmentectomy, lobectomy, sleeve lobectomy) (7-10), robot-assisted surgery has been widely used for standard lobectomy and even complex bronchial surgery such as sleeve lobectomy (11), however, Few reports have investigated robot-assisted surgery used for treatment of glomus tumor. Since the tumor rarely involved the lung parenchyma, lung-reserved technique was usually performed to prevent from pulmonary resection such as sleeve resection and endoscopic removal. In order to minimize surgical trauma, we presented robot-assisted surgery to resect the tumor, this procedure could obtain greater accuracy, precision, and safety as well as minimally invasive. Although endoscopic approach could resect the tumor with less invasive especially when the tumor is tiny, the residual tumor is unclear (12), robotic surgery could achieved thorough resection of tumor under intraoperative frozen section analysis which may be one of merits for its clinical use. 
Management of nourishing blood vessels is important because the blood supply of glomus tumor was extremely rich, ligating and severing the nourishing blood vessels before resecting the tumor was important. In this case, the nourishing blood vessels of tumor were mainly from bronchial artery and were ligated first, so the intra-operative blood loss was less than $100 \mathrm{~mL}$.

Longitudinal resection with transverse suture was also important in this case. Since the tumor involved the majority of airway diameter, appropriate method of suture is important. If the suture direction was perpendicular to the vertical axis of the resected wound (bronchial lumen), it was likely to cause luminal stenosis. In our approach, the suture direction was parallel to the vertical axis of the resected wound (bronchial lumen), this could effectively avoid bronchial stenosis. In this case, 3-0 braided absorbable suture was used for interrupted suture after continuous suture with a 4-0 monofilament nonabsorbable suture, this method was used to reduce the anastomotic tension in order to prevent anastomotic leakage.

In this case, the tumor was radical resected with lungreserved technique under robot-assisted surgery, no complication was found during follow-up, however, our report was only one case, the size or location of tumor may influence the choice of surgical approach. Application of robotic surgery for bronchial glomus tumor still need more clinical evidence, and may applied under certain conditions. In addition, our method (longitudinal resection with transverse suture) may be only used in specific position tumor.

\section{Conclusions}

In conclusion, robot-assisted lung-reserved surgery could be used for treatment of bronchial glomus tumor safely and effectively, longitudinal resection with transverse suture could be one effectively suture method to avoid bronchial stenosis.

\section{Acknowledgments}

Funding: None.

\section{Footnote}

Reporting Checklist: The authors have completed the CARE reporting checklist. Available at https://shc.amegroups.com/ article/view/10.21037/shc-21-16/rc

Peer Review File: Available at https://shc.amegroups.com/ article/view/10.21037/shc-21-16/prf

Conflicts of Interest: Both authors have completed the ICMJE uniform disclosure form (available at https://shc.amegroups. com/article/view/10.21037/shc-21-16/coif). The authors have no conflicts of interest to declare.

Ethical Statement: The authors are accountable for all aspects of the work in ensuring that questions related to the accuracy or integrity of any part of the work are appropriately investigated and resolved. All procedures performed in studies involving human participants were in accordance with the ethical standards of the institutional and/or national research committee(s) and with the Helsinki Declaration (as revised in 2013). Written informed consent was obtained from the patient for publication of this Case report and any accompanying images. A copy of the written consent is available for review by the editorial office of this journal.

Open Access Statement: This is an Open Access article distributed in accordance with the Creative Commons Attribution-NonCommercial-NoDerivs 4.0 International License (CC BY-NC-ND 4.0), which permits the noncommercial replication and distribution of the article with the strict proviso that no changes or edits are made and the original work is properly cited (including links to both the formal publication through the relevant DOI and the license). See: https://creativecommons.org/licenses/by-nc-nd/4.0/.

\section{References}

1. Shugart RR, Soule EH, Johnson EW Jr. Glomus tumor. Surg Gynecol Obstet 1963;117:334-40.

2. Gaertner EM, Steinberg DM, Huber M, et al. Pulmonary and mediastinal glomus tumors--report of five cases including a pulmonary glomangiosarcoma: a clinicopathologic study with literature review. Am J Surg Pathol 2000;24:1105-14.

3. Ravenna F, Saturni S, Casalini A, et al. Bronchial glomus tumor mimicking a COPD exacerbation. Monaldi Arch Chest Dis 2011;75:194-8.

4. Vailati P, Bigliazzi C, Casoni G, et al. Endoscopic removal of a right main bronchus glomus tumor. Monaldi Arch Chest Dis 2004;61:117-9. 
5. Wu HH, Jao YT, Wu MH. Glomus tumor of the trachea managed by spiral tracheoplasty. Am J Case Rep 2014;15:459-65.

6. Sarsam OM, Dunning J, Pochulu B, et al. Robot-assisted bronchoplasty using continuous barbed sutures. J Vis Surg 2018;4:3.

7. Akata S, Yoshimura M, Park J, et al. Glomus tumor of the left main bronchus. Lung Cancer 2008;60:132-5.

8. Takahashi N, Oizumi H, Yanagawa N, et al. A bronchial glomus tumor surgically treated with segmental resection. Interact Cardiovasc Thorac Surg 2006;5:258-60.

9. Azevedo-Pereira AE, Rigueiro MP, Abrão FC. Bronchial

doi: $10.21037 /$ shc-21-16

Cite this article as: Hao Z, Zhang N. Robot-assisted lungreserved bronchial glomus tumor resection: a case report. Shanghai Chest 2022;6:7. glomus tumor with right upper lobe atelectasis. J Bras Pneumol 2010;36:390-3.

10. Lang-Lazdunski L, Bille A, Cane P, et al. Glomus tumour: a rare differential diagnosis of bronchial obstruction in a smoker. Gen Thorac Cardiovasc Surg 2012;60:774-6.

11. Jiao W, Zhao Y, Qiu T, et al. Robotic Bronchial Sleeve Lobectomy for Central Lung Tumors: Technique and Outcome. Ann Thorac Surg 2019;108:211-8.

12. Nakajima E, Takashi H, Taira O, et al. Glomus Tumor in a Segmental Bronchus: A Case Report. Ann Thorac Cardiovasc Surg 2021;27:407-9. 